\title{
On Universal Cycles of Labeled Graphs
}

\author{
Greg Brockman \\ HARVARD UNIVERSITY \\ CAmbridge, MA 02138 \\ United STATES \\ brockman@hcs.harvard.edu \\ Bill Kay \\ University of South Carolina \\ Columbia, SC 29208 \\ United STATES \\ kayw@mailbox.sc.edu \\ Emma E. Snively \\ Rose-Hulman Institute of Technology \\ Terre HaUte, IN 47803 \\ UNited STATES \\ snivelee@rose-hulman.edu
}

Submitted: Aug 26, 2008; Accepted: Dec 14, 2009; Published: Jan 5, 2010

Mathematics Subject Classification: 05C30

\begin{abstract}
A universal cycle is a compact listing of a class of combinatorial objects. In this paper, we prove the existence of universal cycles of classes of labeled graphs, including simple graphs, trees, graphs with $m$ edges, graphs with loops, graphs with multiple edges (with up to $m$ duplications of each edge), directed graphs, hypergraphs, and $k$-uniform hypergraphs.
\end{abstract}

\section{Introduction}

A simple example of a universal cycle (U-cycle) is the cyclic string 11101000, which contains every 3-letter word on a binary alphabet precisely once. We obtain these words by taking substrings of length 3 ; it is useful to imagine that we are looking at the string through a "window" of length 3 , and we shift the window to transition from one word to the next, allowing the window to wrap if necessary.

Universal cycles have been shown to exist for words of any length and for any alphabet size. (For the special case of a binary alphabet, such strings are also known as de Bruijn cycles). The concept easily lends itself to extension, and universal cycles for permutations, partitions, and certain classes of functions are well-studied in the literature (see Chung, Diaconis, Graham [1] for an overview of previous work in the field). In all cases, the distinguishing feature of a universal cycle is that by shifting a window through a cyclic 
string (or in some generalizations, an array), all objects in a given class are represented precisely once.

In this paper we generalize the notion of universal cycles. In particular, we show that these cycles exist for certain classes of labeled graphs. In order to define a universal cycle of graphs, we must first extend the notion of a "window."

Definition 1.1. Given a labeled graph $G$ having vertex set $V(G)=\left\{v_{1}, v_{2}, \ldots, v_{n}\right\}$ with vertices labeled by the rule $v_{j} \mapsto j$ and an integer $0 \leqslant k \leqslant n$, define a $k$-window of $G$ to be the subgraph of $G$ induced by the vertex set $V=\left\{v_{i}, v_{i+1}, \ldots, v_{i+k-1}\right\}$ for some $i$, where vertex subscripts are reduced modulo $n$ as appropriate, and vertices are relabeled such that $v_{i} \mapsto 1, v_{i+1} \mapsto 2, \ldots, v_{i+k-1} \mapsto k$. For each value of $i$ such that $1 \leqslant i \leqslant n$, we denote the corresponding $i^{\text {th }} k$-window of $G$ as $W_{G, k}(i)$. If $G$ is clear from context, we abbreviate our window as $W_{k}(i)$.

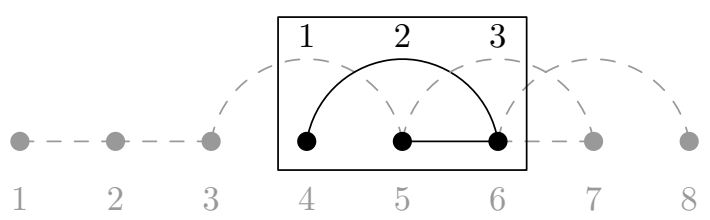

Figure 1 - A 3-window of an 8 vertex graph.

Definition 1.2. Given $\mathcal{F}$, a family of labeled graphs on $k$ vertices, a universal cycle (Ucycle) of $\mathcal{F}$, is a labeled graph $G$ such that the sequence of $k$-windows of $G$ contains each graph in $\mathcal{F}$ precisely once. That is, $\left\{W_{k}(i) \mid 1 \leqslant i \leqslant n\right\}=\mathcal{F}$, and $W_{k}(i)=W_{k}(j) \Longrightarrow i=$ $j$. (Note that the vertex set of the $k$-windows and the elements of $\mathcal{F}$ may be different, however, we will set two labeled graphs equal if they differ only by a bijection between their vertex sets.)

Example 1.3. Note that the full 8 vertex graph in Figure 1 is a U-cycle of simple labeled graphs (graphs without loops or multiple edges) on 3 vertices.

\section{Universal cycles of simple labeled graphs}

We begin our investigation by considering only simple graphs; that is, those without loops or multiple edges. Our result will be that U-cycles of simple labeled graphs on $k$ vertices exist for all $k \geqslant 0, k \neq 2$.

Our proof employs two common notions from the study of U-cycles: the transition graph and arc digraph. The transition graph $T$ of a family $\mathcal{F}$ of combinatorial objects is a directed graph with vertex set $V(T)=\mathcal{F}$. If $A, B \in \mathcal{F}$, there is an edge from $A$ to $B$ in $T$ if and only if $B$ can follow $A$ in one window shift of a $U$-cycle. If $\mathcal{F}$ is a family of graphs on $k$ vertices, this means that the subgraph induced by the vertices labeled $2,3, \ldots, k$ in $A$ is equal to that induced by the vertices $1,2, \ldots, k-1$ in $B$. It should be clear that a 

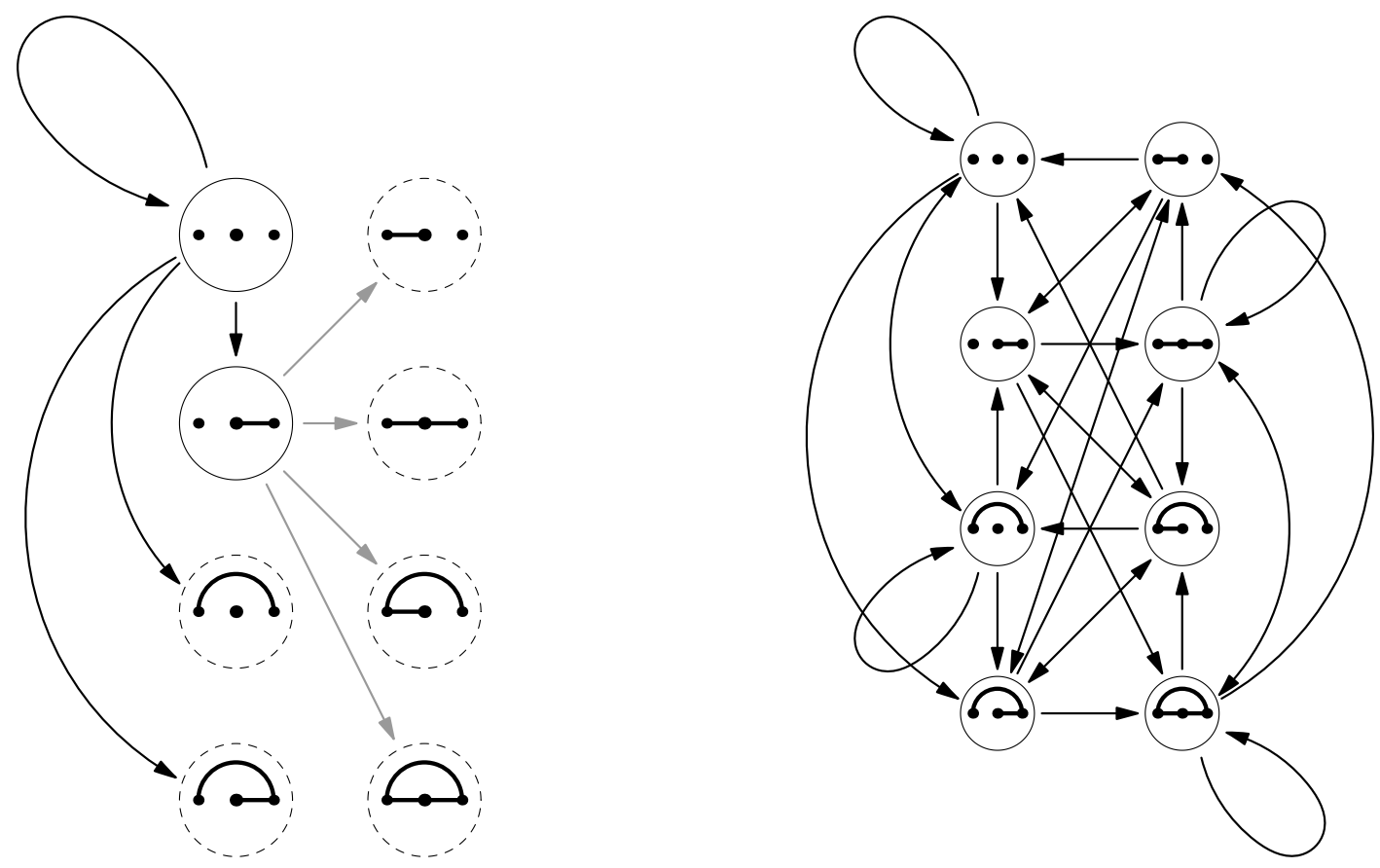

Figure 2 - (Left) A partial sketch of the transition graph of simple graphs on 3 vertices, and (right) the full transition graph. We provide the left figure for clarity.

U-cycle of $\mathcal{F}$ corresponds to a Hamiltonian circuit in $T$ (a directed cycle passing through every vertex exactly once).

Unfortunately, finding Hamiltonian circuits in graphs is an NP-hard problem; however, in our case the problem can be further reduced. Let $D$ be the graph with $E(D)=\mathcal{F}$ such that two edges $A, B$ in $D$ are consecutive (the head of $A$ equals the tail of $B$ ) if and only if $B$ can follow $A$ in a U-cycle. Note that $V(D)$ is arbitrary. Call $D$ the arc digraph of $\mathcal{F}$. Now finding a U-cycle of $\mathcal{F}$ is equivalent to finding an Eulerian circuit in $D$ (a directed cycle passing through every edge exactly once); such circuits are easy to detect. In particular, a graph has an Eulerian circuit if and only if each of its vertices has equal in-degree and out-degree and the graph is strongly connected (for any two vertices $x, y$, there is a directed path from $x$ to $y$ ). For convenience, we often choose the vertices in $V(D)$ to be equal to the "overlap" between consecutive edges. In the following, we construct the transition graph only as a guide for constructing the arc digraph.

Lemma 2.1. The arc digraph $D$ of simple labeled graphs on $k$ vertices, $k \geqslant 3$, has the following properties:

1. For each $\mathcal{X} \in V(D)$, the in-degree of $\mathcal{X}$ equals the out-degree of $\mathcal{X}$.

2. The graph $D$ is strongly connected (there is a directed path from $\mathcal{X}$ to $\mathcal{Y}$ for any $\mathcal{X} \neq \mathcal{Y} \in V(D))$.

Proof. Fix $k \geqslant 3$. Let $\mathcal{F}$ be the set of simple graphs on $k$ vertices. We begin by constructing the transition graph of $\mathcal{F}$. The vertices of this graph are the elements of $\mathcal{F}$. As 


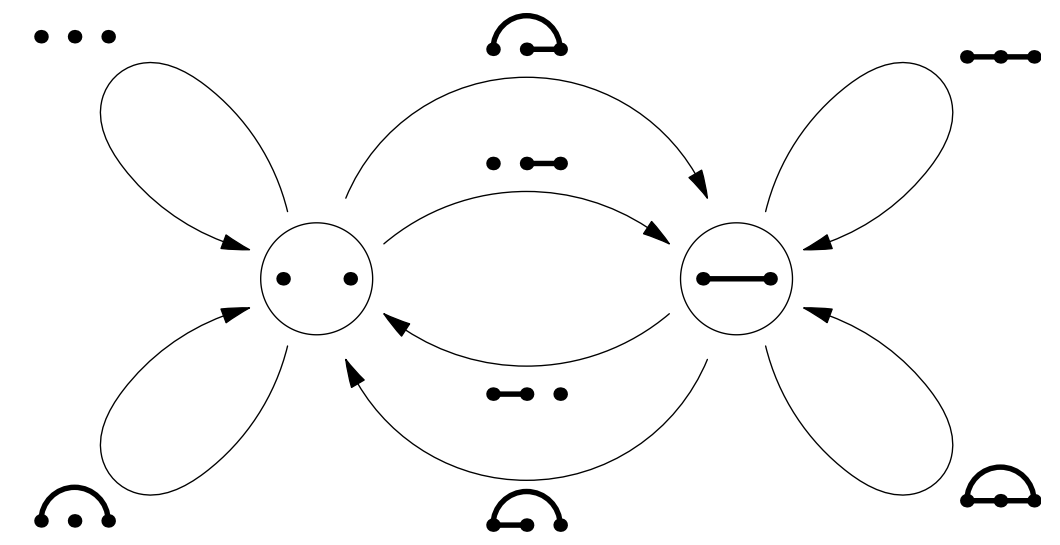

Figure 3 - The arc digraph of the set of simple labeled graphs on 3 vertices.

an example, Figure 2 contains the transition graph for the case $k=3$.

Consider $A, B \in \mathcal{F}$. Let $u$ be the subgraph induced by the vertices labeled $2,3, \ldots, k$ in $A$ (with its vertices relabeled to $1,2, \ldots, k-1$, preserving order) and let $v$ be the subgraph induced by the vertices labeled $1,2, \ldots, k-1$ in $B$. We draw an edge from $A$ to $B$ if and only if $B$ can follow $A$ in a U-cycle, which is equivalent to $u=v$. Thus the transition graph has an edge from $A$ to $B$ if and only if removing the first vertex from $A$ yields the same graph as removing the last vertex from $B$.

We now construct the arc digraph corresponding to this transition graph. Its edge set will be the vertex set of our transition graph. In accordance with the convention mentioned earlier, we use as its vertex set the set of graphs on $k-1$ vertices. By the previous paragraph, the head of an edge $A \in \mathcal{F}$ is the vertex equal to the induced subgraph resulting from removing $A$ 's first vertex. Similarly its tail is the vertex equal to the induced subgraph resulting from removing its last vertex. See Figure 3 for the arc digraph in the case $k=3$.

Proof of 1: Let $\mathcal{X} \in V(D)$ be a vertex in the arc digraph. Since an edge $A \in E(D)$ points into $\mathcal{X}$ if and only if removing the first vertex of $E(D)$ yields $\mathcal{X}$, the in-degree of $\mathcal{X}$ must equal $2^{k-1}$, since the first vertex can arbitarily be adjacent to or not adjacent to each vertex in $V(\mathcal{X})$. Similarly, the out-degree of $\mathcal{X}$ is also $2^{k-1}$ since an edge $B \in E(D)$ points out of $\mathcal{X}$ precisely when deleting its last vertex yields $\mathcal{X}$, and again we have two choices for each vertex in $V(D)$.

Proof of 2: Consider any two vertices of $D, \mathcal{X}$ and $\mathcal{Y}$. Let $G$ be the (labeled) disjoint union of $\mathcal{X}$ and $\mathcal{Y}$, after incrementing the label on each of $\mathcal{Y}$ 's vertices by $k$, as exemplified in Figure 4. Now consider the sequence of $k$-vertex graphs $W_{G, k}(1), W_{G, k}(2), \ldots, W_{G, k}(k+$ 1). Deleting the first vertex of $W_{G, k}(i)$ yields $W_{G, k-1}(i+1)$, as does deleting the last vertex of $W_{G, k}(i+1)$. Thus $W_{G, k}(i)$ and $W_{G, k}(i+1)$ are consecutive in $D$. Furthermore, $W_{G, k}(1)=\mathcal{X}$ and $W_{G, k}(k+1)=\mathcal{Y}$, and hence there is a path in $D$ from $\mathcal{X}$ to $\mathcal{Y}$, as desired.

Theorem 2.2. For each $k \geqslant 0, k \neq 2$, there exists a universal cycle of simple labeled graphs on $k$ vertices. 


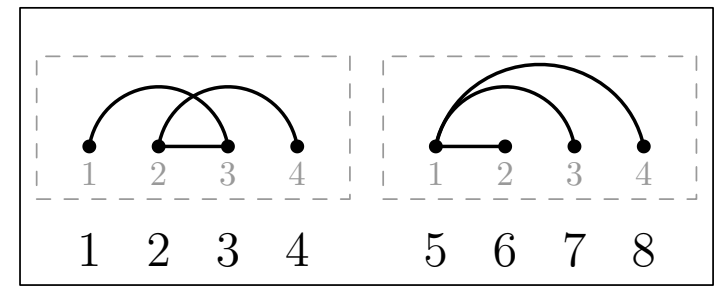

Figure 4 - An example of taking the labeled disjoint union of $\mathcal{X}$ and $\mathcal{Y}$, where each of $\mathcal{X}$ and $\mathcal{Y}$ are graphs on four vertices.

Proof. When $k=0$ or $k=1$ the result is trivial. For $k \geqslant 3$, Lemma 2.1 implies that the arc digraph of simple labeled graphs on $k$ vertices has an Eulerian cycle, and hence a U-cycle of them exists.

Note that for $k=2$ we can modify our definition of a window in order to recognize two distinct windows on two vertices, as shown in Figure 5. Also notice that our proof reduces the problem of finding graph U-cycles to the problem of finding Eulerian cycles of directed graphs, and linear-time algorithms for constructing Eulerian cycles are known in the literature.

\section{General Strategies}

The results of the previous section can be generalized to many classes of graphs, as we show here. Throughout this section, we suppose that all graphs in a given family have $k$ vertices for some fixed $k$. Since our results will equally well apply to hypergraphs, we will consider hypergraphs to be a class of graphs.

Definition 3.1. Let $\operatorname{rot}(X)$ be the rotation class of $X$, or the set of labeled graphs that differ from $X$ only by a cyclic rotation of vertex labels.

Lemma 3.2. Let $\mathcal{F}$ be a family of labeled graphs (which we permit to contain non-simple graphs or even hypergraphs) such that if $X \in \mathcal{F}$, then $\operatorname{rot}(X) \subseteq \mathcal{F}$. Then in the arc digraph of $\mathcal{F}$, for every vertex $V$, the in-degree of $V$ equals the out-degree of $V$.

Proof. Let $V$ be a vertex of the arc digraph of $\mathcal{F}$. Let the set of edges pointing into $V$ be denoted by $I(V)$, and let the set of edges leaving $V$ be denoted $O(V)$. We provide a bijection $f: I(V) \longrightarrow O(V)$, thus proving our lemma. Let $I$ be an edge pointing into $V$
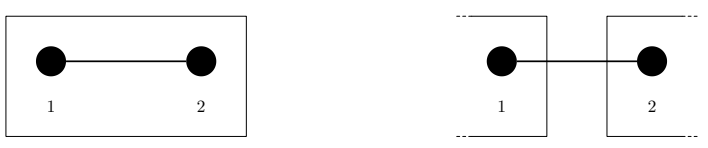

Figure 5 - An illustration of a U-cycle using a modified window for $k=2$. The left window is the complete graph while the right window is the empty graph. Note that an edge is considered to be in a window only if it is not "cut" by the window. 


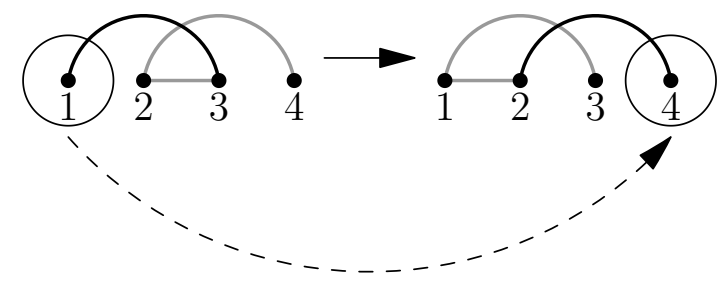

Figure 6 - The cyclic relabeling of the vertices to create an isomorphic graph

(recall that edges in our arc digraph are elements of $\mathcal{F}$ ). If $I$ has $k$ vertices, define $f(I)$ as the graph obtained by cyclically relabeling $I$ as follows: $1 \mapsto k, 2 \mapsto 1,3 \mapsto 2, \ldots, k \mapsto$ $k-1$. Then we see that $f(I) \in \mathcal{F}$, since $f(I)$ is a rotation of $I$, and furthermore $f(I)$ is an edge leaving $V$.

Injectivity of $f$ is clear. Now consider any edge $J$ leaving $V$. Let $I$ be the graph obtained by cyclically relabeling $J$ as follows: $1 \mapsto 2,2 \mapsto 3, \ldots, k-1 \mapsto k, k \mapsto 1$. Again, $I$ is isomorphic to $J$, so $I \in \mathcal{F}$. Furthermore, $I$ is an edge pointing into $V$. Thus $J$ has a preimage under $f$, and $f$ is surjective.

Lemma 3.2 implies that if some class $\mathcal{F}$ of labeled graphs is closed under rotation, then in order to show that a U-cycle of $\mathcal{F}$ exists we need only show that the arc digraph is strongly connected (save for isolated vertices). That is, we must show that given two edges $I$ and $J$ in the arc digraph, there exists a directed path in the arc digraph beginning with $I$ and ending with $J$. In terms of U-cycles, this is equivalent to showing the existence of a graph $G$ such that $W_{G, k}(i)=I, W_{G, k}(j)=J$ and $W_{G, k}(h) \in \mathcal{F}$ for $i \leqslant h \leqslant j$. Or alternatively, we can picture walking on the arc digraph from $I$ to $J$, taking a series of "moves" along consecutive edges, always following the directed arrows.

We now apply these ideas to prove the existence of U-cycles of various classes of graphs.

Theorem 3.3. For each $k \neq 2$, U-cycles exist for the following classes of graphs on $k$ vertices: graphs with loops, graphs with multiple edges (with up to $m$ duplications of each edge), directed graphs, hypergraphs, and $j$-uniform hypergraphs.

Proof. The cases $k=0,1$ are trivial. If $k \geqslant 3$, we proceed in analogy to Part 2 of Lemma 2.1. Take $\mathcal{F}$ to be any of the desired classes of graphs. Pick two graphs $I$ and $J$ from $\mathcal{F}$. Let $G$ be the labeled disjoint union of $I$ and $J$. The graph $I$ is the first $k$-window of $G$, and the graph $J$ is the $(k+1)^{\text {st }} k$-window. Further, each $k$-window $W_{G, k}(i), 1 \leqslant i \leqslant k+1$, is a graph in $\mathcal{F}$. Thus these $k$-windows represent a series of legal edge moves in our arc digraph.

The extensions from Theorem 3.3 followed readily because the relevant graph classes were unrestricted; connectedness of the arc digraph was trivial. Notice that our proof also applies to some restricted classes of graphs, such as forests. We now turn our attention to U-cycles of two types of restricted classes of simple graphs on $k$ vertices.

Theorem 3.4. U-cycles exist for trees on $k$ vertices for $k \geqslant 3$. 
Proof. Let $I, J$ be trees. Let $G$ be the labeled disjoint union of $I$ and $J$. As we read the $k$-windows starting from $I$, let $M$ be the first non-tree window that we arrive upon. Define $v_{M}$ to be vertex of highest label in $M$. Since none of $G$ 's subgraphs contain cycles, we see that there must be one or more components of $M$ that are not connected to the component of $v_{M}$. Draw edges from $v_{M}$ to each of these components; note that the resulting window is now a tree. Furthermore, these edges did not create any cycles in any of $G$ 's $k$-windows, since there are no edges between vertices with label higher than that of $v_{M}$ and those with lower label. Also note that still $W_{G, k}(1)=I, W_{G, k}(k+1)=J$. We then iterate this process until we arrive at a graph that gives us a sequence of $k$-windows, all of which are trees, starting at $I$ and ending at $J$.

Theorem 3.5. U-cycles exist for graphs with precisely $m$ edges.

Proof. For any graph $G$, let $d(G)$ be the degree sequence of $G$. For two graphs $G, H$ having $m$ edges, define $d(G)<d(H)$ if $d(G)$ comes before $d(H)$ lexicographically. We show that for any graph $I$ having exactly $m$ edges, there is a sequence of moves that takes $I$ to the (unique) graph $L$ having $m$ edges and having least degree sequence of graphs with $m$ edges. Thereupon, using the bijection we created in Lemma 3.2, for any graph $J$ having exactly $m$ edges we can reverse its path to $L$ to arrive at a path from $L$ to $J$. This will complete our proof.

Let $I$ be a graph with $m$ edges. If $I$ has least degree sequence, we are done. Otherwise, let $d(I)=\left(d_{1}, d_{2}, \ldots, d_{k}\right)$ and $d(L)=\left(L_{1}, L_{2}, \ldots, L_{k}\right)$. Since $d(I)$ is not minimal, there must be some $i$ such that $d_{i}>L_{i}$. But then there must exist some $j>i$ such that $d_{j}<L_{j}$. Now consider a sequence of moves where at each step we rotate I's vertices according to the relabeling $1 \mapsto k, 2 \mapsto 1, \ldots, k \mapsto k-1$ until the vertex formerly labeled $i$ attains label 1 ; let $i^{\prime}$ be any vertex adjacent to it. Rotate the vertex set once more, but this time connect the vertex formerly labeled $i^{\prime}$ to $j$ instead of $i$. After rotating all of the vertices back to their original positions, we obtain a graph with a smaller degree sequence than $I$. By infinite descent, we see that we can eventually move to $L$ via a sequence of moves all of which are graphs having $m$ edges, thus completing the proof of the theorem.

\section{Conclusions and Future Directions}

In this paper, we have presented a beginning theory of universal cycles of graphs. We have shown the existence of U-cycles of various classes of labeled graphs on $k$ vertices, including simple graphs, multigraphs, graphs on $m$ edges, directed graphs, trees, hypergraphs, and $k$-uniform hypergraphs.

Our work in this field is far from complete. There exist many other classes of graphs for which there conceivably exist U-cycles. However, perhaps the most obvious gap is results regarding U-cycles of unlabeled graphs. The canonical result would be to prove the existence of U-cycles of isomorphism classes of graphs. In such a U-cycle, no two $k$-windows are isomorphic and every isomorphism class is represented as a $k$-window. It is easy to find a U-cycle of isomorphism classes of graphs on 3 vertices. It is difficult, but 
still possible, to find a U-cycle of isomorphism classes of graphs on 4 vertices; one such cycle in exhibited in Figure 7 . These results lead us to conjecture the following.

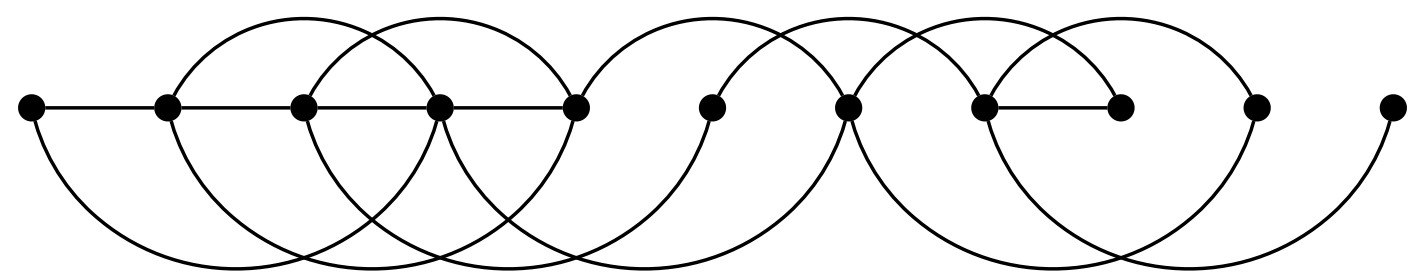

Figure 7 - A U-cycle of isomorphism classes of graphs on 4 vertices.

Conjecture 4.1. For each $k \neq 2$, there exists a $U$-cycle of isomorphism classes of graphs on $k$ vertices.

We also note that U-cycles have potential in theorem-proving as well, as demonstrated by the following result.

Definition 4.2. We say that an integer-valued graph theoretic function $f$ is windowLipschitz if, for all graphs $G$ and $H$ which are one window shift apart in a U-cycle, $|f(G)-f(H)| \leqslant 1$.

Some examples of window-Lipschitz functions are chromatic number and largest clique.

Lemma 4.3. Let $U$ be a $U$-cycle of some family $\mathcal{F}$ of graphs, and let $f$ be a windowLipschitz function defined on these graphs. Then for each integer $\min _{G \in \mathcal{F}} f(G)<i<$ $\max _{G \in \mathcal{F}} f(G)$ there exist at least two distinct elements of $G \in \mathcal{F}$ such that $f(G)=i$.

Proof. By definition of a Lipschitz function, under a single window shift the value of $f$ can change by at most 1 . Hence during the sequence of window shifts from the graph with minimal $f$-value to maximal, every possible value of $f$ in between the minimum and maximum is attained. Similarly, during the sequence of window shifts from the graph with maximal $f$-value to that with minimal, every possible value of $f$ is again attained. This completes our proof.

Finally, we note that it is possible to reduce finding a U-cycle of a set of labeled graphs to finding a U-cycle of an appropriately defined set of equivalence classes of words. For example, let $\mathcal{F}$ be the set of simple labeled graphs on $k$ vertices, and let $\mathcal{G}$ be the set of words of length $k-1$ on the alphabet $\left\{0,1, \ldots, 2^{k-1}-1\right\}$. Define $f: \mathcal{G} \rightarrow \mathcal{F}$ such that $f\left(x_{1} x_{2} \ldots x_{k-1}\right)$ is the graph where, for $1 \leqslant i<j \leqslant k$, there is an edge from $i$ to $j$ if and only if the $j$ th bit of $x_{i}$ is 1 . Now define two words in $\mathcal{G}$ to be equivalent when their image under $f$ is equal, and define the bijection $f^{\prime}$ to map an equivalence class to the image under $f$ of any member of that equivalence class. For an example, see Figure 8.

It is not hard to show that a U-cycle of these equivalence classes (a string whose sequence of $(k-1)$-windows contains exactly one representative from each equivalence class) exists precisely when a U-cycle of $\mathcal{F}$ exists. This reduction allows one to think of U-cycles of graphs in the more traditional context of U-cycles of a restricted class of words. Similar reductions apply to other classes of labeled graphs. 


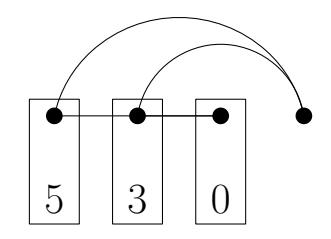

Figure 8 - A graph and an element of its corresponding equivalence class of words, 530 .

\section{Acknowledgements}

This work was done at the East Tennessee State University REU, NSF grant 0552730, under the supervision of Dr. Anant Godbole.

\section{References}

[1] P. Diaconis F. Chung and R. Graham, Universal cycles for combinatorial structures, Discrete Mathematics 110 (1992), 43-59. 